\title{
Polarization and temperature dependence of photoluminescence from zincblende and wurtzite InP nanowires
}

\author{
A. Mishra, L. V. Titova, T. B. Hoang, H. E. Jackson, and L. M. Smith ${ }^{\text {a) }}$ \\ Department of Physics, University of Cincinnati, Cincinnati, Ohio 45221-0011, USA \\ J. M. Yarrison-Rice \\ Department of Physics, Miami University, Oxford, Ohio 45056, USA \\ Y. Kim \\ Department of Physics, College of Natural Science, Dong-A University, Hadan 840, Sahagu, \\ Busan 604-714, Korea \\ H. J. Joyce, Q. Gao, H. H. Tan, and C. Jagadish \\ Department of Electronic Materials Engineering, Research School of Physical Sciences and Engineering, \\ Australian National University, Canberra, Australian Capital Territory 0200, Australia
}

(Received 4 October 2007; accepted 4 December 2007; published online 26 December 2007)

\begin{abstract}
We use polarization-resolved and temperature-dependent photoluminescence of single zincblende (ZB) (cubic) and wurtzite (WZ) (hexagonal) InP nanowires to probe differences in selection rules and bandgaps between these two semiconductor nanostructures. The WZ nanowires exhibit a bandgap $80 \mathrm{meV}$ higher in energy than the $\mathrm{ZB}$ nanowires. The temperature dependence of the PL is similar but not identical for the $\mathrm{WZ}$ and $\mathrm{ZB}$ nanowires. We find that ZB nanowires exhibit strong polarization parallel to the nanowire axis, while the $\mathrm{WZ}$ nanowires exhibit polarized emission perpendicular to the nanowire axis. This behavior is interpreted in terms of the different selection rules for WZ and ZB crystal structures. (C) 2007 American Institute of Physics.
\end{abstract}

[DOI: $10.1063 / 1.2828034]$

The large surface-to-volume ratio of semiconductor nanowires has a profound effect on the energetics of nanowire growth and can result in new structures not observed in the bulk. For instance, only recently has it been understood that one-dimensional growth of InP nanowires (NWs) often occurs preferentially in the hexagonal wurtzite phase, in contrast to the cubic zincblende structure ubiquitous in bulk InP. ${ }^{1-3}$ Indeed, one can use the growth temperature to select between one phase and another. ${ }^{1}$ Such a dramatic structural change should also be reflected by changes in the energy gap, the electron and hole masses, and recombination selection rules. ${ }^{1-3}$ In this letter, we report polarized photoluminescence measurements as a function of temperature from zincblende and wurtzite single InP nanowires.

InP NWs were grown using the gold catalyst-assisted metal-organic vapor-liquid-solid growth mechanism. Undoped InP substrates oriented along the (111) $B$ direction were functionalized by immersion in poly-L-lysine (PLL) solution, rinsed in de-ionized water, and treated with a colloidal solution of monodisperse $30 \mathrm{~nm}$ Au nanoparticles. NWs were grown by horizontal flow metalorganic chemical vapor deposition (MOCVD) at a pressure of $100 \mathrm{mbar}$, with a V/III ratio of 110 . The growth temperature was set at either $42{ }^{\circ} \mathrm{C}$ (zincblende) or $48^{\circ} \mathrm{C}$ (wurtzite). Structural analysis by Transmission Electron Microscopy (Ref. 4) shows that the $42{ }^{\circ} \mathrm{C}$ grown NW resulted in a zincblende (ZB) crystal structure with the growth axis along the $[\overline{1} \overline{1} 1]$ direction. ${ }^{4}$ The ZB NWs were 2-7 $\mu \mathrm{m}$ long with an average diameter of $80 \mathrm{~nm}$. InP NWs grown at $48{ }^{\circ} \mathrm{C}$, in contrast, resulted in $\sim 1 \mu \mathrm{m}$ long tapered NWs which exhibit hexagonal wurtzite (WZ) crystal structure with the growth axis along the [0001] (c

\footnotetext{
${ }^{a)}$ Electronic mail: smithl@physics.uc.edu.
}

axis). For single NW measurements, NWs from the growth substrate were sonicated into Methanol solution and dispersed onto a patterned silicon substrate. The NWs were surveyed using a standard optical microscope to record the positions of $\sim 20 \mathrm{NWs}$ with respect to alignment marks. This substrate was then mounted on the cold finger of a continuous-flow liquid Helium cryostat. A $0.5 \mathrm{~mW}, 780 \mathrm{~nm}$ $\mathrm{CW}$ Ti-sapphire laser was defocused to $>10 \mu \mathrm{m}$ to fully illuminate a single NW. Photoluminescence (PL) from a single NW was collected using a $50 \times / 0.5 \mathrm{NA}$ long working distance microscope objective and a $250 \times$ image of the NW was projected onto the entrance slit of the spectrometer. A matched set of Glan-Thompson linear polarizers and Babinet-Soleil compensators were used both to control the polarization of the laser excitation and to analyze the polarization of the emitted PL.

In Fig. 1, we show PL spectra from three ZB (labeled A, $\mathrm{B}$, and C) and three WZ (labeled 1, 2, and 3) InP nanowires

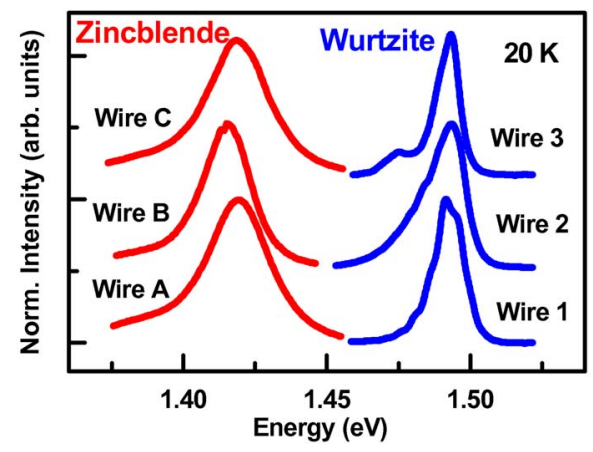

FIG. 1. (Color online) Typical PL spectra from several single ZB and WZ InP nanowires at $15 \mathrm{~K}$. 


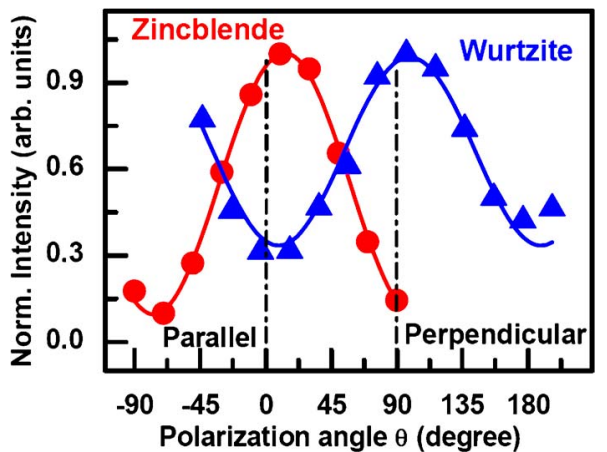

FIG. 2. (Color online) Integrated intensity of PL emission at $15 \mathrm{~K}$ from single ZB NW A and WZ NW 1 as a function of linear polarization analyzer angle.

measured at $15 \mathrm{~K}$. The ZB InP (100) epilayer exhibits a strong and narrow emission line centered at the free exciton recombination energy of $1.418 \mathrm{eV}^{5}$ The three $\mathrm{ZB}$ NWs all display a single broad [full width at half maximum (FWHM) $\sim 20 \mathrm{meV}]$ PL peak which is approximately centered at $1.418 \mathrm{eV}$. As described in Titova et al., while the NW PL from the same growth substrate varies substantially from wire to wire, ${ }^{4}$ the most intense emission is observed for NWs whose PL is centered at $1.418 \mathrm{eV}$ and these are the ones displayed in Fig. 1. These ZB NWs also display lifetimes of $500 \mathrm{ps}-1 \mathrm{~ns}$, lifetimes which are comparable to that observed in the ZB epilayer $(1.7 \mathrm{~ns}){ }^{4}$ The emission width and energy position is strongly affected by state filling and so it is important to measure the NW at the lowest possible excitation intensities. ${ }^{4}$

In contrast, the WZ InP NWs (labeled 1, 2, and 3) display a somewhat narrower emission line (FWHM $\sim 17 \mathrm{meV}$ ) at $1.49 \mathrm{eV}, 80 \mathrm{meV}$ higher than for $\mathrm{ZB}$ InP NWs. Existing published work on the bandgap in WZ InP nanowires has been inconsistent. The $80 \mathrm{meV}$ higher energy WZ NW PL in Fig. 1 is consistent with Mattila et al. ${ }^{1}$ In contrast, Tragardh et al. used photocurrent spectroscopy of $\mathrm{In}_{x} \mathrm{As}_{1-x} \mathrm{P}$ ( $x$ ranging from $0 \%$ to $50 \%$ ) segments inside an InAs nanowire, to estimate that the $\mathrm{WZ}$ InP bandgap ranged from 90 to $230 \mathrm{meV}$ above the ZB InP gap. ${ }^{2,3}$ More recently, Reitzenstein et al. observed multiple emission lines from single WZ InP nanowires in a lower energy range of 1.44-1.47 eV. ${ }^{6}$ Such sharp lower energy emission lines might result from exciton localization at defects as was seen recently in CdS NWs. ${ }^{7}$

The polarization of the PL emission from $\mathrm{ZB}$ and $\mathrm{WZ}$ nanowires also display distinctive differences. To measure this, circularly polarized $780 \mathrm{~nm}$ laser excitation was used to excite all the linearly polarized electronic states with equal probability, while the emitted PL was analyzed for linear polarization. In Fig. 2, the circles show the variation of the PL integrated intensity from $\mathrm{ZB} \mathrm{NW} A$ as a function of the polarization angle, with the solid line a fit to $\cos ^{2} \theta$. The ZB InP NW emission is strongly polarized PL (82\%) parallel to the nanowire axis, ${ }^{8-10}$ due to the strong dielectric contrast between the approximately cylindrical NWs and the surrounding air. In bulk $\mathrm{InP}$, the recombination in $\mathrm{ZB} \mathrm{InP}$ is between the $s$-like conduction band electrons which has $\Gamma_{6}$ symmetry and the $p$-like doubly degenerate light and heavy hole bands with $\Gamma_{8}$ symmetry, and so the emitted light is completely unpolarized. $^{11,12} \mathrm{ZB} \mathrm{NW}$ emission is polarized because of the dielectric contrast. This was also the case in Downloaded 24 Feb 2008 to 150.203 .178 .41 . Redistribution subject

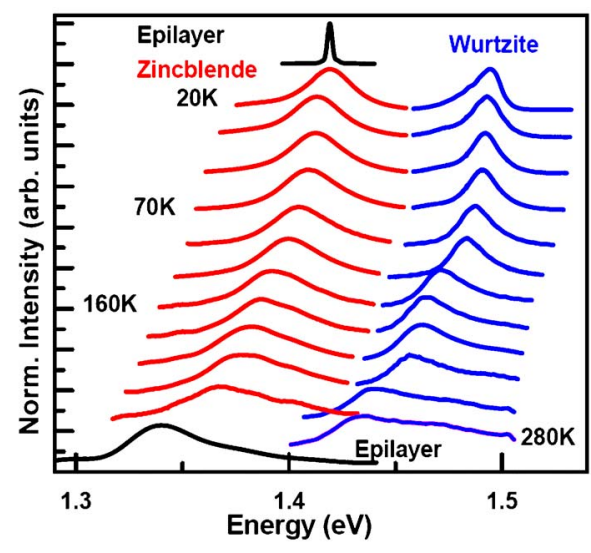

FIG. 3. (Color online) PL spectra from single ZB NW A and WZ NW 1 as a function of temperature. For comparison, PL spectra from a thick (100)oriented epilayer is shown at 15 and $300 \mathrm{~K}$.

previous room temperature measurements of PL in WZ CdSe NWs, which showed the polarization to be determined by dielectric contrast only. ${ }^{13}$ Quantum confinement plays no role since the exciton Bohr radius $(9 \mathrm{~nm})$ in $\mathrm{ZB}$ InP is significantly smaller then the diameter of the NWs $(80 \mathrm{~nm})$.

In contrast, a hexagonal wurtzite crystal $\left(C_{6 v}\right.$ point group) exhibits a completely different symmetry. The $s$-like conduction band has $\Gamma_{7}$ symmetry, while the $p$-like hole bands split into three separate hole bands due to a combination of the spin-orbit interaction and crystal field splitting. ${ }^{11,12}$ The lowest energy hole states (highest lying energy bands) having a symmetry of $\Gamma_{9}$ (the so-called $A$ band), while the two higher energy hole bands ( $B$ and $C$ bands) have $\Gamma_{7}$ symmetry. ${ }^{11,12}$ The lowest energy exciton state should be between a $\Gamma_{7}$ electron and a $\Gamma_{9}$ hole, which means that the $\Gamma_{7} \rightarrow \Gamma_{9}$ recombination is dipole allowed only for $E$ perpendicular to the $c$ axis of the nanowire, and forbidden for $E$ parallel to the $c$ axis. ${ }^{11,12}$ Thus the lowest energy exciton transition in WZ InP should be strongly polarized perpendicular to the wurtzite $c$ axis and, thus, perpendicular to the nanowire axis.

Polarization analysis of the emission from wurtzite NW 1 shows that the PL is polarized (49\%) perpendicular to the NW axis, consistent with growth of the NW along the [0001] axis. The degree of polarization in the $\mathrm{WZ}$ nanowire is weaker than $\mathrm{ZB}$ nanowire because the dipole selection rules of the wurtzite crystal are modulated by the dielectric contrast inherent for all semiconductor NWs.

In Fig. 3, we show the variation of the PL emission from ZB NW A and WZ NW 1 as a function of temperature from 15 to $280 \mathrm{~K}$ and compare this with PL emission from the epilayer at 15 and $300 \mathrm{~K}$. Emission from both ZB and WZ NWs shifts monotonically to lower energy with increasing temperature, and the linewidths increase markedly. The intensity of the PL becomes weaker, but never completely quenches. In Fig. 4(a), we plot emission energy versus temperature for both the $\mathrm{ZB}$ and $\mathrm{WZ}$ NWs, along with a $\mathrm{ZB}$ $2 \mu \mathrm{m}$ thick epilayer. The solid lines are fits to the modified Varshni equation $E=E_{0}-\alpha T^{4} /\left(T^{3}+\beta\right) .{ }^{14}$ The emission energy is comparatively flat at low temperatures, but decreases almost linearly at temperatures above $50 \mathrm{~K}$. Interestingly, the WZ NWs appear to follow most closely the temperature dependence of the epilayer, though shifted up by $80 \mathrm{meV}$ in energy. In contrast, the ZB NW at high temperatures exhibits to AIP license or copyright; see http://apl.aip.org/apl/copyright.jsp 

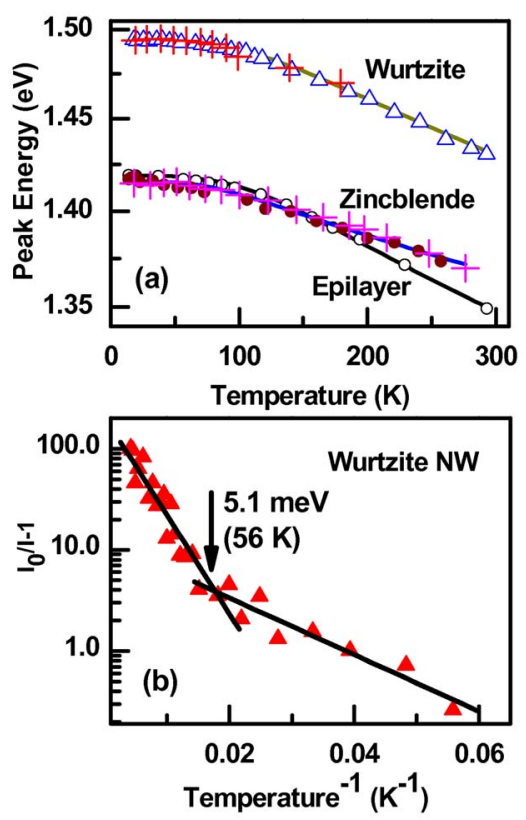

FIG. 4. (Color online) (a) Peak position of the emitted PL as a function of temperature for WZ NWs $1(\mathbf{\Delta})$ and $2(+)$ and ZB NWs A $(\bullet)$ and B (+). For comparison, the peak position of the emitted PL from a thick (100)oriented InP epilayer is shown $(O)$. (b) The integrated intensity $I$ of WZ NW 1 is plotted as $I_{0} / I-1$ as a function of $1 / T$, where $I_{0}$ is related to the integrated intensity at zero temperature. The exciton binding energy of $5.1 \mathrm{meV}$ is marked by arrow.

a significantly different slope compared to the bulk InP epilayer. The reason for this difference is presently unclear.

In Fig. 4(b), we plot $I_{0} / I-1$, where $I_{0}$ reflects the low $T$ PL intensity, and $I$ is the integrated intensity of the PL emission from the WZ InP nanowire. ${ }^{15}$ The fact that the PL can be easily measured at room temperature indicates a low nonradiative surface recombination rate. While the PL becomes somewhat less intense in the temperature range between 15 and $50 \mathrm{~K}$, the PL emission intensity decreases rapidly above $50 \mathrm{~K}$. The data display an abrupt change in slope at $56 \mathrm{~K}$, near the temperature which separates exciton dominated emission at lower temperatures and electron-hole plasma emission at higher temperatures. Temperature-dependent PL data for single $\mathrm{ZB}$ InP nanowires are essentially equivalent, which may indicate that the electron and hole masses are not dramatically different between the ZB and WZ nanowires

To summarize, we have shown that the growth of $Z B$ or WZ InP nanowires can be controlled by changing the growth substrate temperature. Striking changes in emission polarization and energy are observed which reflect the change in symmetry and dipole selection rules. WZ NWs emit $80 \mathrm{meV}$ higher energy than do ZB NWs. The variation of the intensity and emission energy with temperature for $\mathrm{ZB}$ and $\mathrm{WZ}$ NW are similar except at high temperatures and apart from the bandgap difference. Importantly, the emission for both $\mathrm{ZB}$ and $\mathrm{WZ}$ nanowires is quite intense without passivation, indicating notable low sensitivity to the surface of these InP NWs.

A.M., L.V.T., T.B.H., H.E.J., L.M.S., and J.M.Y.-R. acknowledge support from the Institute for Nanoscale Science and Technology of the University of Cincinnati and the National Science Foundation through Grant Nos. EEC/NUE 0532495 and ECCS 0701703. The Australian authors acknowledge support from the Australian Research Council. Y.K. acknowledges support by the Korean Science and Engineering Foundation (KOSEF) through Grant No. F012007-000-10087-0.

${ }^{1}$ M. Mattila, T. Hakkarainen, M. Mulot, and H. Lipsanen, Nanotechnology 17, 1580 (2006).

${ }^{2}$ J. Taagardh, A. I. Persson, J. B. Wagner, D. Hessman, and L. Samuelson, J. Appl. Phys. 101, 123701 (2007).

${ }^{3}$ H. Pettersson, J. Tragardh, A. I. Persson, L. Landin, D. Hessman, and L. Samuelson, Nano Lett. 6, 4 (2006).

${ }^{4}$ L. V. Titova, T. B. Hoang, J. M. Yarrison-Rice, H. E. Jackson, Y. Kim, H. J. Joyce, Q. Gao, H. H. Tan, C. Jagadish, X. Zhang, J. Zou, and L. M. Smith, Nano Lett. 7, 3383 (2007).

${ }^{5}$ D. C. Reynolds, C. W. Litton, R. J. Almassy, S. B. Nam, P. J. Dean, and R. C. Clarke, Phys. Rev. B 13, 2507 (1976).

${ }^{6}$ S. Reitzenstein, S. Munch, C. Hofmann, A. Forchel, S. Crankshaw, L. C. Chuang, M. Moewe, and C. Chang-Hasnain, Appl. Phys. Lett. 91, 091103 (2007).

${ }^{7}$ L. V. Titova, T. B. Hoang, H. E. Jackson, L. M. Smith, J. M. Yarrison-Rice, J. L. Lensch, and L. J. Lauhon, Appl. Phys. Lett. 89, 053119 (2006).

${ }^{8}$ J. F. Wang, M. S. Gudiksen, X. F. Duan, Y. Cui, and C. M. Lieber, Science 293, 1455 (2001).

${ }^{9}$ H. E. Ruda and A. Shik, Phys. Rev. B 72, 115308 (2005).

${ }^{10}$ T. B. Hoang, L. V. Titova, J. M. Yarrison-Rice, H. E. Jackson, A. O. Govorov, Y. Kim, H. J. Joyce, H. H. Tan, C. Jagadish, and L. M. Smith, Nano Lett. 7, 588 (2007).

${ }^{11}$ J. L. Birman, Phys. Rev. 114, 1490 (1959).

${ }^{12}$ J. L. Birman, Phys. Rev. Lett. 2, 157 (1959).

${ }^{13}$ C. X. Shan, Z. Liu, and S. K. Hark, Phys. Rev. B 74, 153402 (2006).

${ }^{14}$ M. Cardona, T. A. Meyer, and M. L. W. Thewalt, Phys. Rev. Lett. 92, 196403 (2004).

${ }^{15}$ A. Chiari, M. Colocci, F. Fermi, Y. H. Li, R. Querzoli, A. Vinattieri, and W. H. Zhuang, Phys. Status Solidi B 147, 421 (1988). 